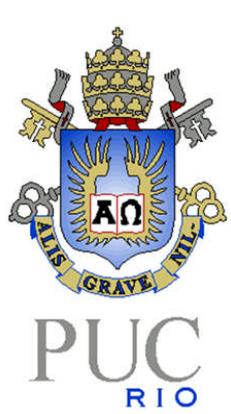

Rodrigo Klim Gomes

\title{
A DESSALINIZAÇÃO TÉRMICA COMO ALTERNATIVA PARA ABASTECIMENTO DE ÁGUA \\ Estudo da Técnica de Dessalinização Térmica e Avaliação \\ Econômica Preliminar
}

Dissertaçao de Mestrado

Dissertação apresentada ao programa de PósGraduação em Engenharia Mecânica da PUC-Rio como requisito parcial para obtenção do título de Mestre em Engenharia Mecânica.

Orientador: Prof. Carlos Valois Maciel Braga 


\section{Rodrigo Klim Gomes}

\section{A DESSALINIZAÇÃO TÉRMICA COMO ALTERNATIVA PARA ABASTECIMENTO DE ÁGUA Estudo da Técnica de Dessalinização Térmica e Avaliação Econômica Preliminar

\begin{abstract}
Dissertação apresentada ao programa de PósGraduação em Engenharia Mecânica da PUC-Rio como requisito parcial para obtenção do título de Mestre em Engenharia Mecânica. Aprovado pela comissão examinadora abaixo.
\end{abstract}

Prof. Carlos Valois Maciel Braga

Orientador Departamento de Engenharia Mecânica - PUC-Rio

Prof. Eloi Fernández Y Fernández Departamento de Engenharia Mecânica - PUC-Rio

Prof. Sergio Leal Braga Departamento de Engenharia Mecânica - PUC-Rio

Prof. Luiz Antônio Vaz Pinto Departamento de Engenharia Naval e Oceânica - COPPE/UFRJ

Prof. José Eugênio Leal Coordenador Setorial do Centro Técnico Científico - PUC-Rio 
Todos os direitos reservados. É proibida a reprodução total ou parcial do trabalho sem autorização da universidade, do orientador e do autor.

\section{Rodrigo Klim Gomes}

Graduou-se em Engenharia Naval na UFRJ em 2004, desde então atuou como engenheiro na área offshore adquirindo relativa experiência prática. Concluiu MBA em Gerenciamento de Projetos pela Fundação Getúlio Vargas em 2010 com trabalho final voltado para desenvolvimento esportivo de crianças de escolas municipais do Rio de Janeiro. Publicou artigos nos congressos OMAE, DOT e Rio Pipeline, abordando temas relativos à engenharia naval e engenharia de dutos.

Ficha Catalográfica

Gomes, Rodrigo Klim

A dessalinização térmica como alternativa para abastecimento de água : estudo da técnica de dessalinização térmica e avaliação econômica preliminar / Rodrigo Klim Gomes ; orientador: Carlos Valois Maciel Braga. - 2011.

108 f. : il. (color.) ; $30 \mathrm{~cm}$

Dissertação (mestrado)-Pontifícia Universidade Católica do Rio de Janeiro, Departamento de Engenharia Mecânica, 2011.

Inclui bibliografia

1. Engenharia mecânica - Teses. 2. Dessalinização. 3. Primeira lei da termodinâmica. 4. Viabilidade. I. Braga, Carlos Valois Maciel. II. Pontifícia Universidade Católica do Rio de Janeiro. Departamento de Engenharia Mecânica. III. Título. 
Para minha mãe, a grande responsável por todas as minhas conquistas. 


\section{Agradecimentos}

À PUC-Rio pela concessão da bolsa de isenção, sem a qual a realização do curso não seria possível.

À minha mãe e minha irmã, as pessoas que me apoiam e estimulam em todos os momentos de dificuldade e de felicidade. Ao meu pai pelo exemplo de perseverança.

À minha esposa, o prêmio que o Criador me reservou depois de ter atravessado o deserto.

Ao meu orientador, Carlos Valois Maciel Braga, pela confiança, pela paciência, pela amizade e pelo apoio incondicional. Muito obrigado mestre.

Ao professor Eloi Fernández, o primeiro a ouvir a ideia inicial daquilo que viria a se tornar um trabalho acadêmico. Obrigado pela boa vontade.

Ao professor Luiz Fernando Azevedo, pois sem sua disposição em ajudar a conclusão do curso não seria possível.

Aos amigos Bruno Fontes Rodrigues, pelo companheirismo e pelo estímulo, e John Phillip Bernardes, pelo exemplo.

À INTECSEA do Brasil, pela tolerância e pelo apoio.

Às secretárias Rosely Ribeiro, do DEM, e Sandra Lara, do DAR, pois foram primordiais para que esse momento fosse possível.

A todos os amigos que me inspiraram e estimularam ao longo da caminhada.

Sobretudo, a Deus e ao Mestre Jesus, pela oportunidade de aprimoramento. 


\section{Resumo}

Gomes, Rodrigo Klim; Braga, Carlos Valois Maciel A Dessalinização Térmica como Alternativa para Abastecimento de Água - Estudo da Técnica de Dessalinização Térmica e Avaliação Econômica Preliminar. Rio de Janeiro, 2011, 108p. Dissertação de Mestrado - Departamento de Engenharia Mecânica, Pontifícia Universidade Católica do Rio de Janeiro

A dessalinização térmica é uma técnica utilizada em diversas partes do mundo para produção de água. Países como Estados Unidos, Israel, Espanha e Grécia utilizam esse tipo de sistema em grande escala, garantindo um abastecimento constante para alguns pontos de seu território e contribuindo para o aperfeiçoamento dos sistemas de produção. No Brasil, apesar da vasta bacia hidrográfica e da existência de grandes reservatórios subterrâneos, a dessalinização térmica poderia ser vista como alternativa de produção para apoiar o desenvolvimento agrícola e industrial em algumas regiões, criando também melhores condições sociais para a população beneficiada, especialmente no nordeste brasileiro. Nessa dissertação propomos um método para avaliação preliminar de diversos cenários para implantação de tais sistemas. A avaliação aborda inicialmente a definição do dessalinizador, realizada através das formulações disponíveis em trabalhos acadêmicos que têm como base a Primeira Lei da Termodinâmca. Tais formulações foram adaptadas para o caso em estudo. Em seguida são avaliados os principais parâmetros para viabilidade do projeto, tais como Valor Presente Líquido (VPL) e Índice de Lucratividade (IL), de forma a demonstrar se o projeto seria ou não viável. Para consolidação da metodologia utilizada foi criado um programa que permite a avaliação de diversos cenários de maneira independente, sendo de fácil acesso e de grande flexibilidade para o usuário interessado neste tema.

\section{Palavras-chave}

Dessalinização; Primeira Lei da Termodinâmica; Viabilidade. 


\section{Abstract}

Gomes, Rodrigo Klim; Braga, Carlos Valois Maciel Braga (Advisor). Thermal Desalination as an Option for Water Supply - A Study of Thermal Desalination Technology and Preliminary Economic Feasibility Analysis. Rio de Janeiro, 2011, 108p. Msc. Dissertation - Departamento de Engenharia Mecânica, Pontifícia Universidade Católica do Rio de Janeiro

Thermal desalination is a technology used in many places for water production. Some countries use desalination to produce big volume of water, like USA, Israel, Spain and Greece, aiming for the steady supply of water to some specific regions, contributing to the improvement of production systems. In Brazil, inspite of the huge volume of water available through the rivers, thermal desalination should be considered as an alternative for water production supporting the development of country for food production and for industry, specially in the northeastern region. In this work, a method of evaluation for different water supply needs was conceived, in order to perform the preliminary evaluation of this kind of system. It begins with the definition of the thermal desalinator which will compose the production system. This first step was based on First Law of Thermodynamics formulations available in technical articles used as reference. After technical analysis, the main parameters used for the study of feasibility are defined, for example, the Net Present Value. The methodology extracted from reference articles was simplified and converted into an algorithm, developted for an easy evaluation of different situations.

\section{Keywords}

Desalination; First Law of Thermodynamics; Feasibility. 


\section{Sumário}

1 Introdução 17

1.1. Considerações iniciais 17

1.2. Revisão Bibliográfica $\quad 18$

1.3. Justificativa do Trabalho 21

2 Sistemas de Dessalinização e Aquedutos 24

2.1. Técnicas de Dessalinização 24

2.2. Consumo Energético dos Sistemas de Dessalinização 29

2.3. Custo de Manutenção dos Sistemas de Dessalinização 30

2.4. Nível de Pureza da Água Produzida 31

2.5. Estimativa de Custos dos Sistemas de Dessalinização 31

2.6. Aqueduto 33

3 Metodologia 35

3.1. Visão Geral 35

3.2. Sistema de Dessalinização Térmica 36

3.3. Modelo Simplificado $\quad 45$

3.4. Estimativa de Entalpia 49

3.5. Formulação para Balanço de Massa e Energia 55

3.6. Viabilidade Econômica 63

$\begin{array}{ll}4 \text { Programa Computacional } & 67\end{array}$

4.1. Descrição Geral do Programa $\quad 67$

4.2. Entrada de Dados $\quad 70$

4.3. Resultados $\quad 77$

5 Estudo de Caso $\quad 81$

5.1. Estudo de caso 1 - Indústria de Papel e Celulose 82

5.2. Estudo de caso 2 - Cidada de Santa Bárbara 85

5.3. Estudo de caso 3 - Região Metropolitana do Nordeste 89

5.4. Estudo de caso 4 - Abastecimento de Condomínios 94

5.5. Estudo de caso 5 - Cidade de Guamaré 97

6 Conclusões e Recomendações $\quad 100$

6.1. Aplicabilidade dos Sistemas de Dessalinização no Brasil 100 
6.2. Resultados Obtidos 101

6.3. Sugestões para Trabalhos Futuros 102

7 Referências Bibliográficas 104

8 Apêndice 106 


\section{Lista de Figuras}

Figura 1 - Representação esquemática do processo de osmose. 24

Figura 2 - llustração de uma planta de osmose reversa. 25

Figura 3 - Configuração de sistema de osmose reversa em espiral. 25

Figura 4 - Sistema de dessalinização por osmose reversa; sistema tubular. 26

Figura 5 - llustração do sistema MEE Horizontal. Ilustração obtida em Semiati (2000). $\quad 29$

Figura 6 - Comparação entre os custos dos sistemas de osmose reversa e dessalinização térmica. 32

Figura 7 - Arranjo do sistema. $\quad 35$

Figura 8 - Foto de uma planta de dessalinização chinesa. 37

Figura 9 - Foto de uma planta de dessalinização vertical. 38

Figura 10 - Representação do fluxo de água e vapor dentro de uma célula. 38

Figura 11 - Diagrama esquemático do sistema de dessalinização térmica baseado em Hatzikioseyian (2003). Linha vermelha pontilhada representa o vapor e linha azul representa a água do mar.

Figura 12 - Representação esquemática da câmara de evaporação (flash chamber). 40

Figura 13 - Representação esquemática do evaporador. 41

Figura 14 - Representação esquemática do filtro. 42

Figura 15 - Representação esquemática do pré-aquecedor. 43

Figura 16 - Representação esquemática do misturador de vapor. 44

Figura 17 - Representação esquemática do misturador de condensado. $\quad 44$

Figura 18 - Diagrama esquemático do sistema de dessalinização simplificado com apenas 3 células. $\quad 46$

Figura 19 - Evaporador na célula $1 . \quad 47$

Figura 20 - Pré-aquecedor na célula $1 . \quad 47$

Figura 21 - Evaporador na célula $2 . \quad 47$

Figura 22 - Pré-aquecedor na célula $2 . \quad 48$

Figura 23 - Evaporador na célula $3 . \quad 48$

$\begin{array}{ll}\text { Figura } 24 \text { - Pré-aquecedor na célula } 3 . & 48\end{array}$ 
Figura 25 - Gráfico para estimativa da entalpia do concentrado de água e sal de acordo com Dittman (1977). 50

Figura 26 - Gráfico da IAPWS para diferentes estados. 51

Figura 27- Exemplo de fluxo de caixa. 65

Figura 28 - Fluxo de dados do programa. 68

Figura 29 - Estrutura do módulo 1.68

Figura 30 - Estrutura do módulo 2.69

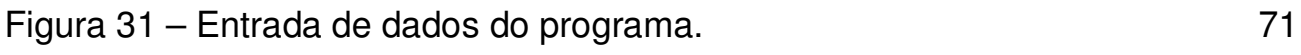

Figura 32 - Dados da área. $\quad 72$

Figura 33 - Entrada de dados para o sistema de dessalinização térmica. $\quad 73$

Figura 34 - Entrada de dados para a planta termoelétrica. 73

Figura 35 - Entrada de dados para sistema de transportes. 74

Figura 36 - Entrada de dados para fatores de projeto do dessalinizador. $\quad 75$

Figura 37 - Entrada de dados para viabilidade econômica. 76

Figura 38 - Tela para visualização de resultado parcial. 76

Figura 39 - Botões de comando do programa. $\quad 77$

Figura 40 - Tela para visualização de resultado parcial. 78

Figura 41 - Configuração do Estudo de caso 1 - Indústria de papel e celulose. 82

Figura 42 - Resultados para estudo de caso 1.86 


\section{Lista de Tabelas}

Tabela 1 - Resultado de entalpia adimensional para as regiões 1 e 2 definidas pelo modelo da IAPWS.

Tabela 2 - Dados gerais para estudo de caso 1.

Tabela 3 - Resultados para estudo de caso 1.

Tabela 4 - Dados gerais para estudo de caso 2.

Tabela 5 - Resultados iniciais para estudo de caso 2.

Tabela 6 - Resultados para estudo de caso 2 com alteração no valor de comercialização.

Tabela 7 - Dados gerais para estudo de caso 3.

Tabela 8 - Resultados para estudo de caso. Erro no cálculo do sistema de dessalinização.

Tabela 9 - Resultados para estudo de caso 3.

Tabela 10 - Dados gerais para estudo de caso 4.

Tabela 11 - Resultados para estudo de caso 4.

96

Tabela 12 - Dados gerais para estudo de caso 5 .

97

Tabela 13 - Resultados para estudo de caso 5. 


\section{Lista de Símbolos}

$\mathrm{H} \quad$ Perda de carga ao longo do duto $(\mathrm{m})$

F Fator de atrito

$\mathrm{L} \quad$ Comprimento do duto (m)

G Aceleração da gravidade $\left(\mathrm{m} / \mathrm{s}^{2}\right)$

$\mathrm{V} \quad$ Velocidade do fluido no interior do duto $(\mathrm{m} / \mathrm{s})$

D Diâmetro do aqueduto $(\mathrm{m})$

K Fator de atrito

Q Vazão do fluido no interior do duto $\left(\mathrm{m}^{3} / \mathrm{s}\right)$

$P \quad$ Massa específica da água $\left(\mathrm{kg} / \mathrm{m}^{3}\right)$

$\mathrm{Z} \quad$ Elevação em relação ao nível do mar $(\mathrm{m})$

A Área da seção interna do duto $\left(\mathrm{m}^{2}\right)$

P Potência de bombeio (W)

$\mathrm{H}$ Eficiência da bomba

m Vazão em massa $(\mathrm{kg} / \mathrm{s})$

$\dot{Q} \quad$ Taxa de transferência de calor $(\mathrm{kW})$

R Constante dos gases $(\mathrm{kJ} / \mathrm{kgK})$

$\mathrm{T} \quad$ Temperatura $(\mathrm{K})$

$\mathrm{T}$ Adimensional de temperatura

$\Pi \quad$ Adimensional de pressão

J Coeficiente para determinação empírica da entalpia

$\mathrm{H} \quad$ Entalpia $(\mathrm{kJ} / \mathrm{kg})$

$\mathrm{P} \quad$ Pressão $(\mathrm{kPa})$

$\Gamma \quad$ Coeficiente obtido para determinação empírica da entalpia

$\mathrm{N} \quad$ Coeficiente para determinação empírica da entalpia

C Concentração da solução ( $\mathrm{kg} \mathrm{sal} / \mathrm{kg}$ solução)

F Fator para determinação das caraterísticas do trocador de calor

VP Valor presente

I Taxa de desconto anual

N Quantidade de anos para amortização do financiamento 


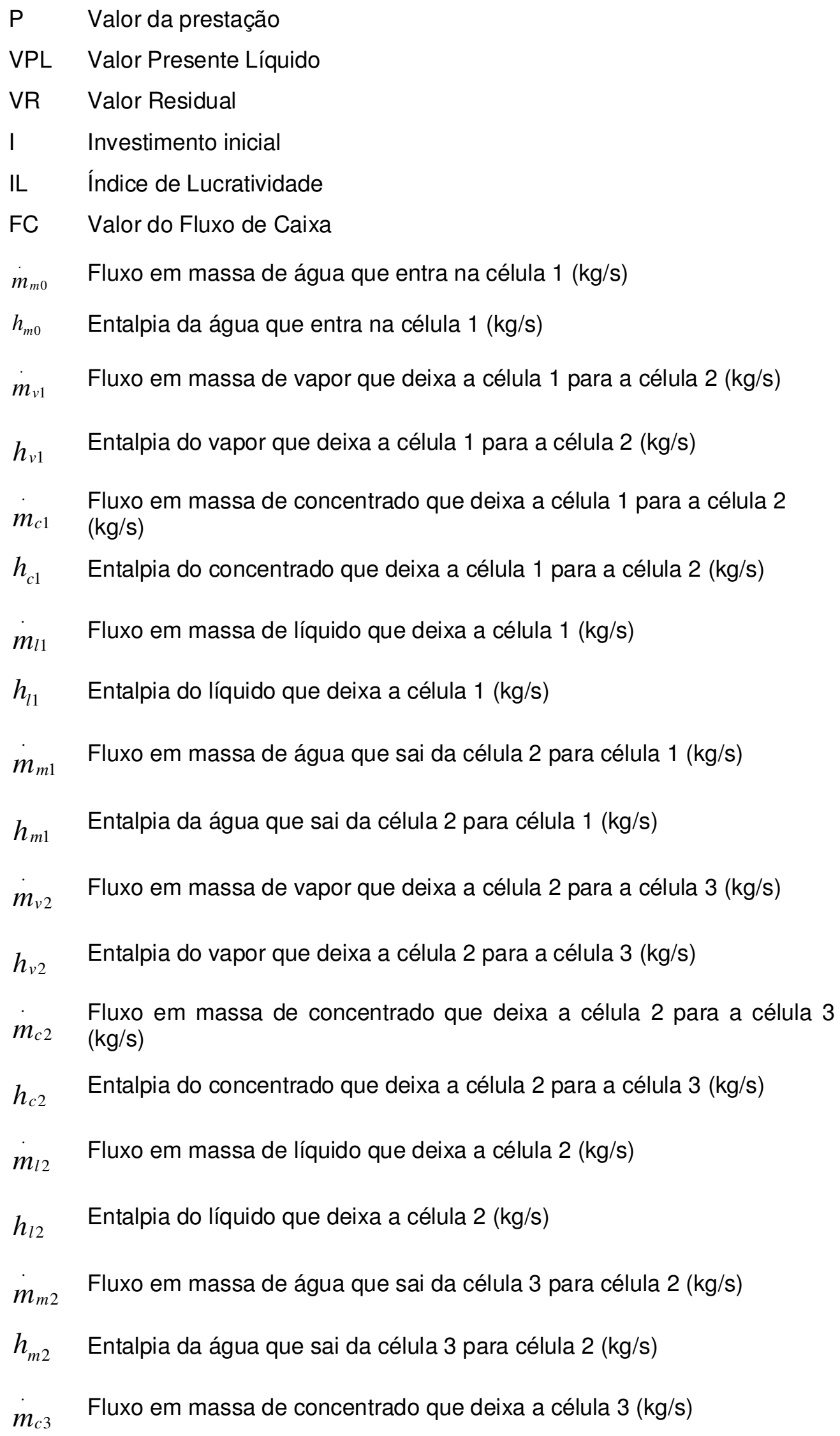


$h_{c 3} \quad$ Entalpia do concentrado que deixa a célula $3(\mathrm{~kg} / \mathrm{s})$

$\dot{m}_{l 3} \quad$ Fluxo em massa de líquido que deixa a célula $3(\mathrm{~kg} / \mathrm{s})$

$h_{l 3} \quad$ Entalpia do líquido que deixa a célula $3(\mathrm{~kg} / \mathrm{s})$

$\dot{m}_{m 3} \quad$ Fluxo em massa de água que entra na célula $3(\mathrm{~kg} / \mathrm{s})$

$h_{m 3} \quad$ Entalpia da massa de água que entra na célula $3(\mathrm{~kg} / \mathrm{s})$

$\dot{m}_{l} \quad$ Fluxo em massa de líquido total que deixa o dessalinizador $(\mathrm{kg} / \mathrm{s})$

$h_{l} \quad$ Entalpia do líquido total que deixa o dessalinizador $(\mathrm{kg} / \mathrm{s})$

- Fluxo em massa de vapor que deixa o evaporador da célula 1 em direção ao pré-aquecedor da célula $1(\mathrm{~kg} / \mathrm{s})$

$h_{v 1} \quad$ Entalpia do vapor que deixa o evaporador da célula 1 em direção ao préaquecedor da célula $1(\mathrm{~kg} / \mathrm{s})$

$\dot{m}_{v 1 \text { '" }} \quad$ Fluxo em massa de vapor que deixa o pré-aquecedor da célula $1(\mathrm{~kg} / \mathrm{s})$

$h_{v 1 \text { "' }} \quad$ Entalpia do vapor que deixa o pré-aquecedor da célula $1(\mathrm{~kg} / \mathrm{s})$

$\dot{m}_{l 1 \text { "' }} \quad$ Fluxo em massa de líquido que deixa o pré-aquecedor da célula $1(\mathrm{~kg} / \mathrm{s})$

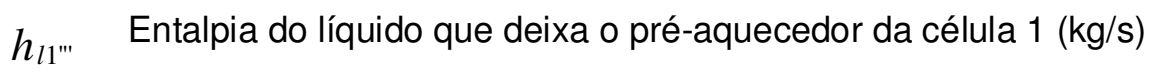

$\dot{m}_{v 1} \quad$ Fluxo em massa de vapor que entra na célula 2 vindo da célula $1(\mathrm{~kg} / \mathrm{s})$

$h_{v 1} \quad$ Entalpia do vapor que entra na célula 2 vindo da célula $1(\mathrm{~kg} / \mathrm{s})$

$\dot{m}_{v 1 "} \quad$ Fluxo em massa de vapor produzido no evaporador da célula $2(\mathrm{~kg} / \mathrm{s})$

$h_{v 1 "} \quad$ Fluxo em massa de vapor produzido no evaporador da célula $2(\mathrm{~kg} / \mathrm{s})$

$\dot{m}_{l 1 "} \quad$ Fluxo em massa de líquido produzido pelo evaporador da célula $2(\mathrm{~kg} / \mathrm{s})$

$h_{l 1 "} \quad$ Entalpia do líquido produzido pelo evaporador da célula $2(\mathrm{~kg} / \mathrm{s})$

$\dot{m}_{v^{\prime}} \quad$ Fluxo em massa de vapor que deixa o evaporador da célula 2 em direção ao pré-aquecedor da célula $2(\mathrm{~kg} / \mathrm{s})$

$h_{v 2^{\prime}} \quad$ Entalpia do vapor que deixa o evaporador da célula 2 em direção ao préaquecedor da célula $2(\mathrm{~kg} / \mathrm{s})$ 
$\dot{m}_{v 3^{\prime}} \quad$ Fluxo em massa de vapor que deixa o evaporador da célula $3(\mathrm{~kg} / \mathrm{s})$

$h_{v 3^{\prime}} \quad$ Entalpia do vapor que deixa o evaporador da célula $3(\mathrm{~kg} / \mathrm{s})$

$\dot{m}_{v 2 "} \quad$ Fluxo em massa de vapor produzido no evaporador da célula $3(\mathrm{~kg} / \mathrm{s})$

$h_{v 2 \text { " }} \quad$ Entalpia do vapor produzido no evaporador da célula $3(\mathrm{~kg} / \mathrm{s})$

$\dot{m}_{l 2 "} \quad$ Fluxo em massa de líquido produzido pelo evaporador da célula $3(\mathrm{~kg} / \mathrm{s})$

$h_{l 2 \text { " }} \quad$ Entalpia do líquido produzido pelo evaporador da célula $3(\mathrm{~kg} / \mathrm{s})$

F Coeficiente para determinação da eficiência do trocador de calor. Dado proveniente do fabricante

$h_{c 1} \quad$ Entalpia do fluxo em massa de concentrado que deixa a célula $1(\mathrm{~kJ} / \mathrm{kg})$

$h_{c 2} \quad$ Entalpia do fluxo em massa de concentrado que deixa a célula $2(\mathrm{~kJ} / \mathrm{kg})$

$h_{c 3} \quad$ Entalpia do fluxo em massa de concentrado que deixa a célula $3(\mathrm{~kJ} / \mathrm{kg})$

$h_{v 2} \quad$ Entalpia do fluxo em massa de vapor que entra no evaporador da célula $3(\mathrm{~kJ} / \mathrm{kg})$

$h_{l 2 "} \quad$ Entalpia do fluxo em massa de líquido que entra no evaporador da célula $3(\mathrm{~kJ} / \mathrm{kg})$ 